\title{
Hybrid Wiener-Hammerstein Structure for Grey-Box Modeling of DC-DC Converters
}

\author{
J. A. Oliver, R. Prieto, J. A. Cobos, O. García and P. Alou
}

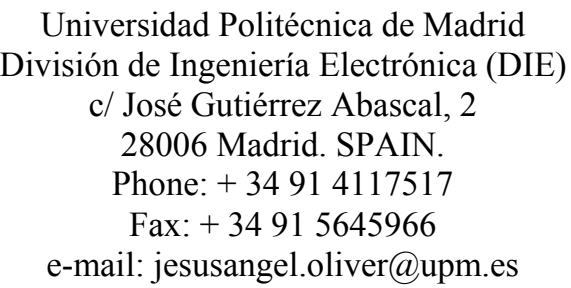

\begin{abstract}
In this work a generic hybrid model for dc-dc converters is proposed. The protections and remote control functions are managed by the logic system (event driven behaviour) and the power stage and control is modelled by a Wiener-Hammerstein structure. The model is oriented to be parameterized based on the information provided by manufacturers in their datasheet and it is able to predict power consumption, efficiency, system stability and large signal behaviour including inrush current, protections, start-up and remote on-off control.
\end{abstract}

\section{INTRODUCTION}

Traditionally, dc-dc converters have been designed ad-hoc for each particular application. Present day commercial dc-dc converters are replacing custom designs because of their significant advantages in terms of development time, they usually have lower area and smaller form factors, and they reduce the component count and also the cost. Additionally, standardization initiatives like POLA (Point of Load Alliance) or DOSA (Distributed-power Open Standard Alliance) supported by most of the manufacturers are increasing the added value of the commercial solutions due to the existence of second sources [1-2]. Figure 1 shows a typical distributed power architecture based on commercial DC-DC converters.

In these cases, the internal structure of the dc-dc converter and their parameters are not known, since they are part of the intellectual property of the manufacturer, and the averaging techniques cannot be applied [3]. So it is necessary to develop new modelling techniques that can capture the behaviour of the converters preferably by means of the information provided by manufacturers in their datasheet. In this way, parameter analysis to determine the robustness of the system can be applied based on actual manufacturers' information.

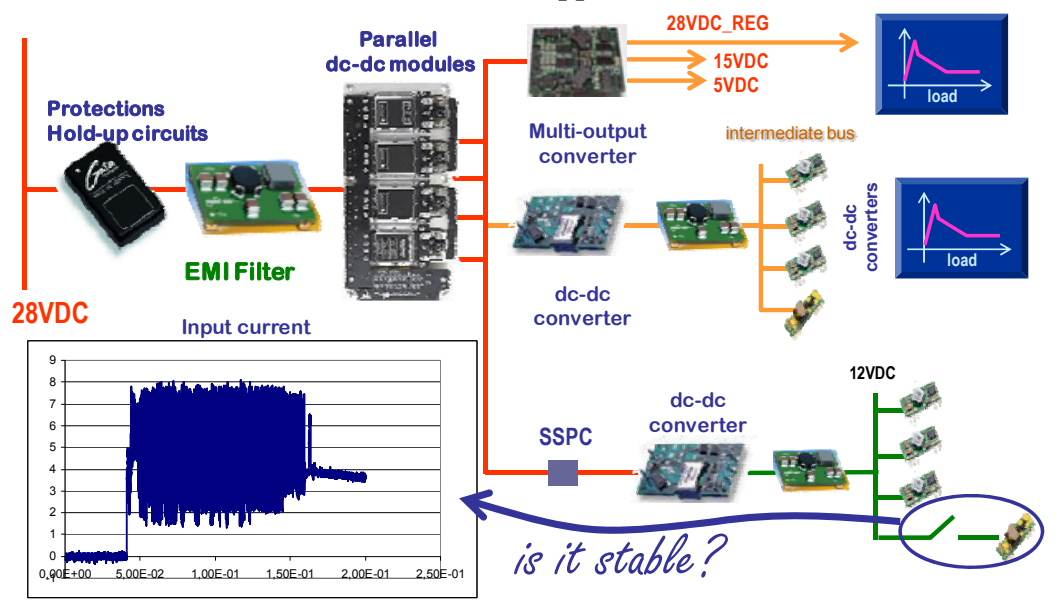

Figure 1 Example of a DC power distribution system for an avionic application

This necessity has been recently identified [3-5]. The modelling approach proposed by [5] is based on the small signal frequency identification of the converter. This approach is very accurate from the point of view of small-signal stability but it does not account for efficiency as a function of the input voltage and load, output voltage as a function of load or protections such as overvoltage, over-current, under-voltage and temperature.

The proposed model overcomes all those limitations. The trade-offs between simulation time and accuracy of dc-dc converters have been successfully addressed by the use of a hybrid model as shown in Figure 2.

\section{PROPOSED HYBRID WIENER- HAMMERSTEIN STRUCTURE}

Control signals, protections and start-up behaviour are extremely important in the assessment of the stability of distributed power systems. These are events that modify the behaviour of the converter and have to be considered. 
Taking into account the hybrid nature of dc-dc converters [6], the model is partitioned in two blocks:

- The logic system (event driven behaviour) that manages all the protections and remote control features.

- Continuous/discrete system that includes the power stage and its control, independently if it is implemented digitally or analogy.

The logic system can be modelled by means of a state diagram and its implementation on a circuit simulator will depend on the modelling capabilities of the simulator. It will measure the analog variables that can modify the behaviour of the dc-dc converter (output voltage, output current, input voltage, temperature, etc.) and, based on its current state and the analog inputs, it will generate the control signal for the power stage and control.

The power stage and control is modelled by means of a Wiener-Hammerstein structure (see Figure 2). This structure has the advantage of gathering all the non-linear static behaviour of the converter, usually provided in datasheets, by means of one block. This non-linear behaviour includes:

- Variation of the output voltage with respect to the output current and input voltage

- Variation of the efficiency with the load and the input current.

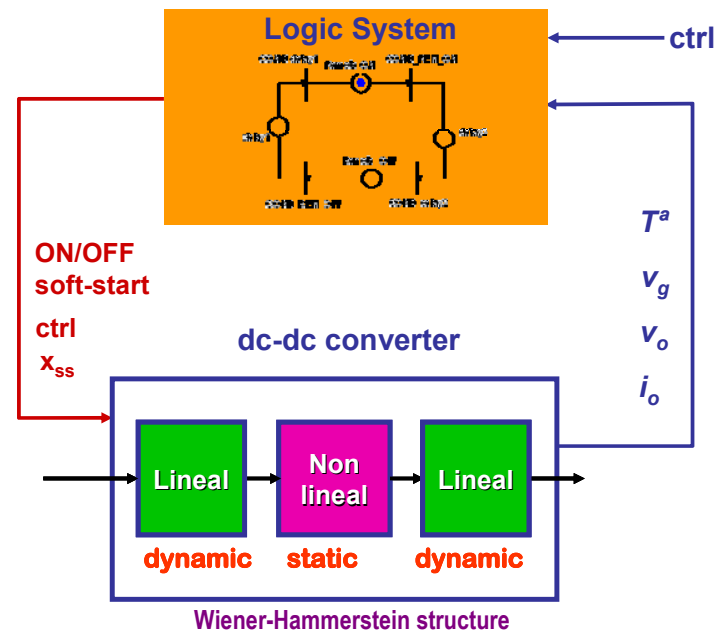

Figure 2 Proposed dc-dc converter model structure

\section{A. Proposed Wiener-Hammerstein structure}

One of the most critical steps in the modelling process is to select the model structure. If the selected structure is wrong, it does not matter what optimization algorithm is used to fit the model that it will never find a correct solution.

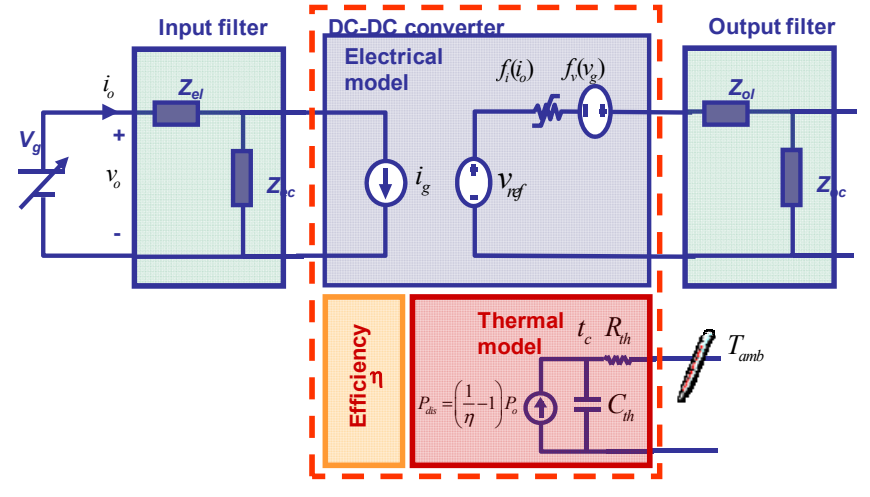

Figure 3 Proposed Wiener-Hammerstein structure for the $d c-d c$ converter model

In this case, the problem is how to find a model structure valid for all kind of DC-DC converters (with and without zeroes in the RHP, hard switched PWM converters or frequency controlled resonant converters). The proposed model for the power stage is based on a Wiener-Hammerstein structure shown in Figure 2. It consists of:

- a non-linear static model that will represent the behaviour of the converter in steady state.

a. Efficiency as a function of input voltage and output current

b. Output voltage as a function of the reference, the input voltage and the output current

- Dynamic input block that will account for the high frequency input impedance behaviour of the converter and its initial inrush current.

- Dynamic output block that will account for the transient behaviour of the converter under load changes.

Additionally, a simple thermal model has been included to account for the effect of thermal protections.

The equations for the static-nonlinear model are given by:

$$
\begin{gathered}
v_{\text {out }}=v_{\text {ref }}+f_{v}\left(v_{\text {in }}\right)-f_{z}\left(i_{\text {out }}\right) \\
i_{\text {in }}=\frac{1}{\eta\left(v_{\text {in }}, i_{\text {out }}\right)} \frac{v_{\text {out }} \cdot i_{\text {out }}}{v_{\text {in }}}
\end{gathered}
$$

The implementation of these equations in a circuit oriented simulator based on the information given by the manufacturers is quite straightforward. The dependence of the output voltage with the input voltage and output current can be implemented by a 2D lookup table, or by means of two 1D lookup tables [3]. This solution in spite of been an approximation provides very good results in terms of accuracy and convergence.

Additionally, the dependence of the efficiency with the input voltage and output current can be also implemented by means of a 2D lookup table or two 1D lookup tables. 


\section{1) Linear dynamic output network}

In order to propose a suitable network to consider the output dynamics of a generic converter it is necessary to establish some assumptions that will be discussed:

1. There is a capacitive element place at the output. Actually, this is always the case to reduce the output voltage ripple and reduce the output impedance. This capacitive element does not need to be ideal and the model can include the equivalent series resistance (ESR) and inductance (ESL) of the capacitors bank.

2. Output voltage of the converter is controlled through the injected current. This is almost true for current mode controlled converters up to the crossover frequency of the current loop. In voltage mode controlled converters seems less obvious but, as it will be shown the approach is also applicable but with higher error.

According to these assumptions the small-signal model of the output stage of a general current controlled converter can be represented as shown in Figure 4, where: $Z_{c}$ is the equivalent impedance of the output capacitors (including parasitics), $K_{v}$ is the output voltage controller transfer function, $\tilde{i}_{c}$ is the injected current, $\tilde{v}_{r e f}$ is the reference voltage and $\tilde{i}_{o}$ is the current demanded by the load.

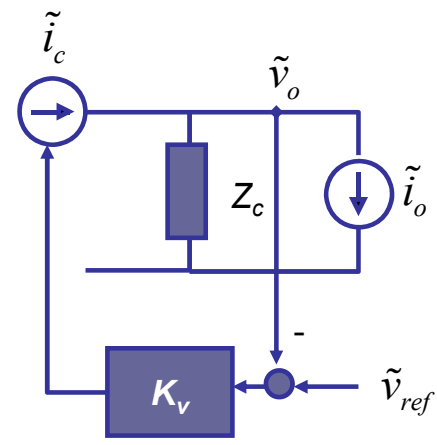

Figure 4 Equivalent small-signal circuit of the output stage of a current controlled converter

According to the equivalent circuit shown in Figure 4, and after some math, it is possible to arrive to the following expression for the output voltage (3):

$v_{o}(s)=-\frac{Z_{C}(s)}{1+Z_{C}(s) \cdot K_{v}(s)} \cdot i_{o}(s)+\frac{Z_{C}(s) \cdot K_{v}(s)}{1+Z_{C}(s) \cdot K_{v}(s)} \cdot v_{r e f}(s)$

This transfer function is also the same as the transfer function of the equivalent circuit of Figure 5, just making the series impedance $Z_{L}$ equal to the inverse of the voltage loop regulator (4):

$$
Z_{L}(s)=\frac{1}{K_{v}(s)}
$$

As a consequence the proposed dynamic network to fit the output dynamics of the converter is the one shown in Figure 5.

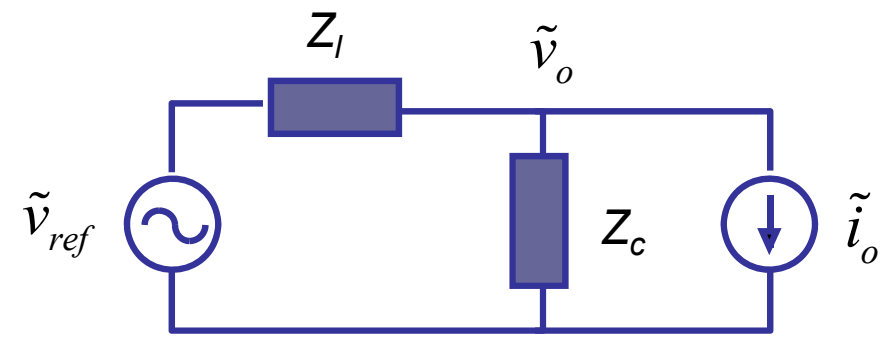

Figure 5 Equivalent small signal circuit of the output stage

Since the output network does not have to change the static behaviour of the converter, which is accounted by the nonlinear network, the condition that has to be satisfied by this network is:

$$
\frac{Z_{L}(0)}{Z_{C}(0)}=0
$$

From the point of view of the parametric identification it is necessary to figure out the structure of the series impedance, $Z_{l}$, to identify its components. In order to do that, the form of the transfer function $K_{v}$ will be analyzed.

According to the assumptions made above and the small signal model shown in Figure 4, the output voltage can be controlled by means of a PI controller:

$$
K_{v}(s)=\frac{\omega_{i}}{s} \cdot\left(1+\frac{s}{\omega_{z}}\right)
$$

where:

- $\omega_{i}$ adjust the crossover frequency and

- $\omega_{z}$ sets the phase margin.

This controller, according to (4) yields to the following equivalent series impedance:

$$
Z_{l}(s)=\frac{1}{K_{v}(s)}=\frac{\frac{1}{\omega_{i}} s}{1+\frac{s}{\omega_{z}}}
$$

The network that fits into this transfer function consists of an inductance in parallel with a resistor of the following values:

$$
\begin{gathered}
L=\frac{1}{\omega_{i}} \\
R_{d}=\frac{\omega_{z}}{\omega_{i}}
\end{gathered}
$$

Applying this procedure to the current controlled converter of Figure 4 with equivalent output capacitors impedance (C, $\mathrm{R}_{\mathrm{ESR}}$ ), the linear output network will have the structure shown in Figure 6. This output network can be easily identified based 
on the current step response data provided by the manufacturer or an equivalent measurement.

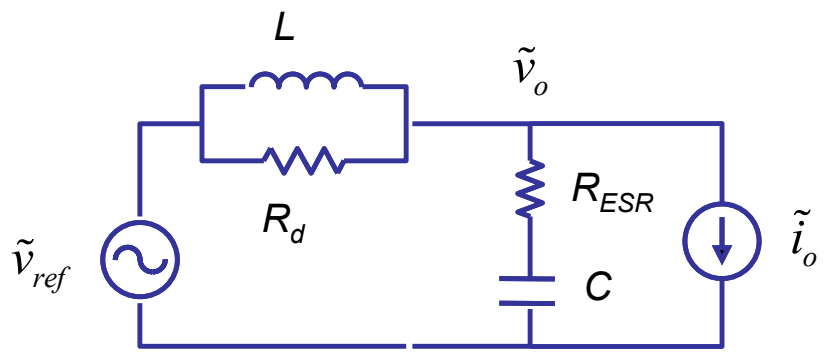

Figure 6 Equivalent closed loop circuit

\section{2) Linear dynamic input network}

The proposed structure of the input network is shown in Figure 3 , named as input filter. The selection of this network is based on the typical configuration of the EMI input filters for dc-dc converters.

This network has to be selected under the constraint of not modifying the static behaviour of the converter, given by the nonlinear static block. As a consequence, it has to satisfy the same condition in steady state as the output network (5).

One possible network that can be fitted based on inrush current data is shown in Figure 7[3-4]. This network is valid for both over-damped and under-damped inrush current behaviour.

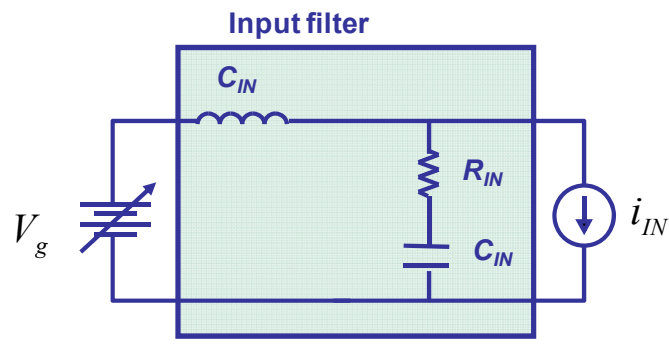

Figure 7 Candidate input filter network

\section{B. Event-driven behaviour}

In this work, most commons events have been included based on a careful search of main dc-dc manufacturers. The events included in this model are:

- Remote control. This signa lis usually provided to dc-dc converters to enable or disable them.

- Input voltage protection. This protection disables the operation of the converter when the input voltage is out of the operation limits of the converter, avoiding its malfunctioning.

- Under-voltage protection (with and without hysteresis). Below this voltage the converter will be turned off to avoid high currents that will destroy it.

○ Over-voltage protection (with and without hysteresis). This voltage assures that all the components of the converter are working with the designed voltage rates.
- Output voltage protection. It is usually an upper output voltage value that will disable the converter to protect it against overvoltage caused by an external source.

- Thermal protection. In the case of high temperature operation of the converter this protection will also turn it off.

- Over-current protection. This protection tries to avoid high internal current due to high external loads. This protection is included inside the event-driven behavior in case of turning-off the converter. The protection based on lowering the output voltage as a function of the output voltage has to be included in the static non linear model since it does not creates an event.

\section{MODEL VALIDATION}

\section{A. Individual converter validation}

The validation of the proposed model has been done over tens of different dc-dc converters with different input voltages, output voltages, power ranges, topologies and controls.

One of the key parameters in the evaluation of a distributed power architecture is the global efficiency as a function of the operating conditions. Most of the electronic systems (data servers, mobile devices, radio base stations, etc.) are operating a significant part of the time under low working conditions. As a consequence the efficiency of the power converters at light load has an important effect on the global performance.

To show how the proposed model fits the measured values of the efficiency as a function of the load conditions the comparison of the measured and simulated results for a Lambda X15-48S05 converter [7] are shown in Figure 8. The match between measurements and simulations is due to the implementation of the efficiency by means of a look-up table that fits all the measured points.

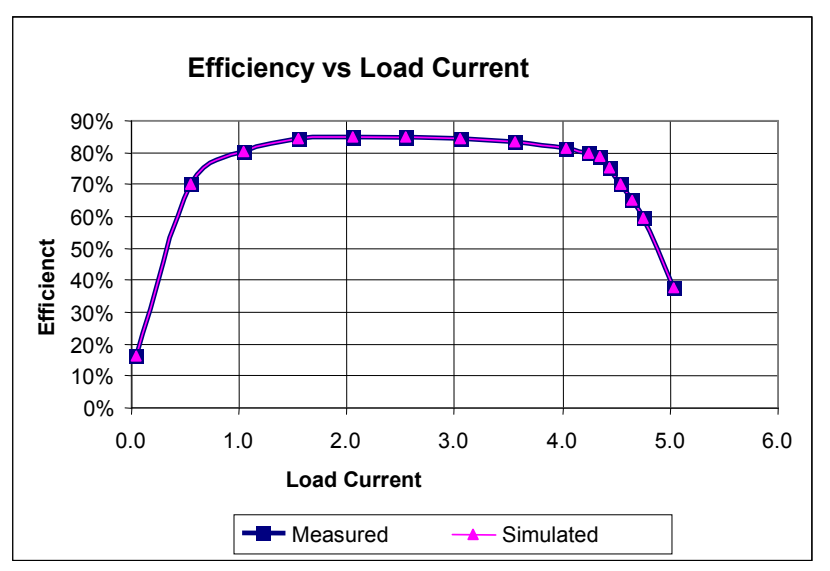

Figure 8 Measured and simulated efficiency vs load current at $48 \mathrm{~V}$

The validation of the event-driven behaviour, the linear dynamic input and output blocks and the static non-linear behaviour working together can be obtained by means of an start-up of the converter under no-load and full load conditions. In this case, the start-up delay, the soft-start, the inrush current and the effect of the load on the behaviour are all taken into account. 
Figure 9 shows the start-up sequence of the converter at full load. It can be seen how the model predicts the inrush current at $1.7 \mathrm{~ms}$, it also accounts for the delay of the turn-on and the soft-start of the converter.

The same experiment has been carried out at light load and the results are shown in Figure 10. In this case it can be also seen that the model accounts for the inrush current, the turn-on delay and the soft-start. It can be also noticed that the effect of the load on the input current is also correctly accounted.

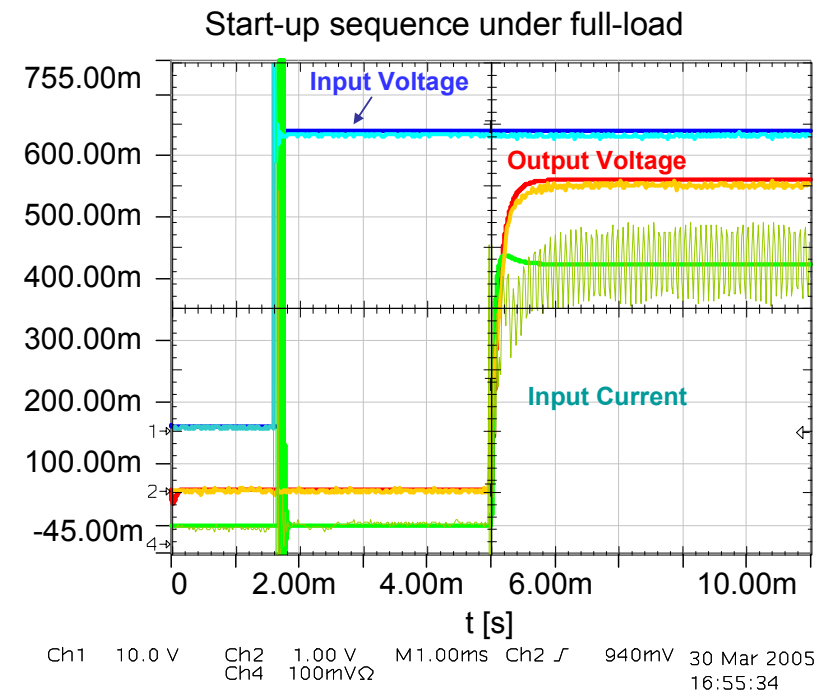

Figure 9 Measured and simulated start-up transient response under full load conditions

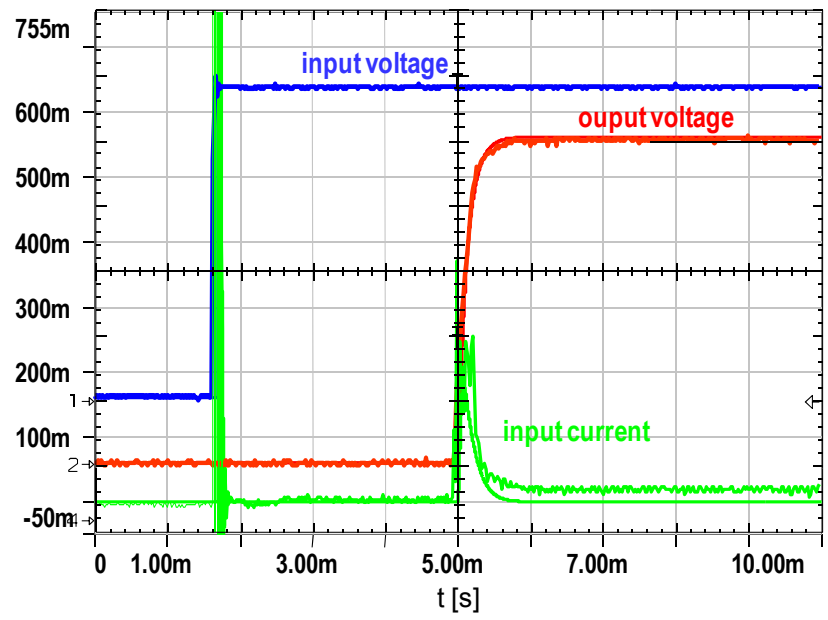

Figure 10 Measured and simulated start-up transient response under no-load conditions

\section{B. System level validation}

The proposed model has also been validated in the simulation of several spacecraft power distribution networks. The results shown in Figure 11 and Figure 12 correspond the start-up current of actual 28VDC distributed power system for an spacecraft application, that consists of eleven commercial dcdc converters, twelve filters, protections, a hold-up circuit and six non-linear loads, under two different bus bar impedance conditions.
Both cases are for minimum input voltage $(20 \mathrm{~V})$, but the bus voltage drop for the first one is set to $0,4 \mathrm{~V}$ and the second one to $1,5 \mathrm{~V}$.

When the drop in the distribution bus is set to $0,4 \mathrm{~V}$, the system is able to start-up without problems. Nevertheless, when the voltage drop in the bus is increased to $1,5 \mathrm{~V}$ the system becomes unstable.

The global stability of the system is also predicted by the simulation employing the models for the dc-dc converters shown in this paper. Especially important is the addition of the event-driven behaviour to the models. In this case the instability of the system cannot be predicted without considering the protection of the converters.

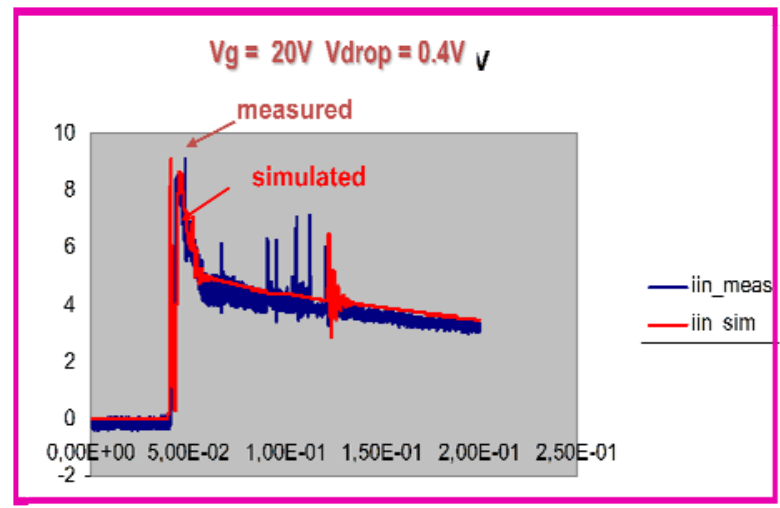

Figure 11 Measurement vs simulated input current comparison for a power-up test with Vdrop $=0,4 \mathrm{~V}$

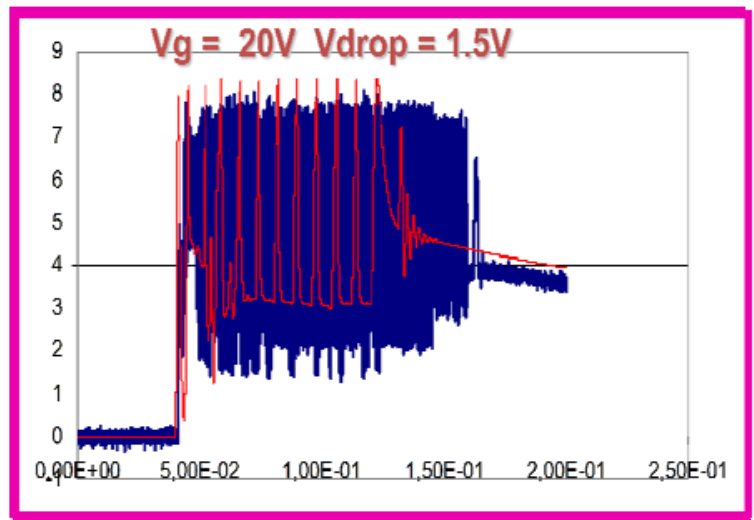

Figure 12 Measurement vs simulated input current comparison for a power-up test with Vdrop $=1,5 \mathrm{~V}$

The stability problem was caused by the interactions between the under-voltage protection of a converter and its input filter. During the start-up, when the input voltage of the converter was higher than the minimum input voltage it started the turnon process, demanding a high input current due to its fast settle time. This current reflected at the input of the converter caused a voltage drop in the input filter that activated the undervoltage protection and so, disabling the converter. When the input voltage of the converter reached again the minimum input voltage the same process repeated again, causing the oscillation. 


\section{CONCLUSIONS}

A generic model structure for DC-DC converters is proposed based on the hybrid nature of the converters. The protections and remote control features are managed by the logic system (event driven behaviour) and the power stage and control is modelled by a Wiener-Hammerstein structure.

A justification of the networks of the linear dynamic input and output networks is also provided based on the typical EMI filter configuration and the output voltage control respectively.

The generic model structure proposed in this paper accounts for:

\begin{tabular}{|l|l|l|}
\hline \multicolumn{1}{|c|}{$\begin{array}{c}\text { Non linear static } \\
\text { model }\end{array}$} & $\begin{array}{c}\text { Linear dynamic } \\
\text { model }\end{array}$ & \multicolumn{1}{|c|}{ Event-driven model } \\
\hline $\begin{array}{l}\text { Efficiency as a } \\
\text { function of load and } \\
\text { input voltage }\end{array}$ & $\begin{array}{l}\text { Output load } \\
\text { dynamics }\end{array}$ & Remote control \\
\hline $\begin{array}{l}\text { Output voltage as } \\
\text { output current and } \\
\text { input voltage }\end{array}$ & $\begin{array}{l}\text { Input voltage } \\
\text { dynamic }\end{array}$ & $\begin{array}{l}\text { Input voltage protection: } \\
\text { - Under-voltage } \\
\text { - Over-voltage }\end{array}$ \\
\hline & Inrush current & $\begin{array}{l}\text { Output voltage protection: } \\
\text { - Short-circuit } \\
\text { - Over-voltage }\end{array}$ \\
\hline & & Temperature protection \\
\hline & &
\end{tabular}

The validation of the model has been carried out both at component level and system level. An the applicability of the model to analyze the performance of dc distributed power systems has also been highlighted.

\section{REFERENCES}

[1] Roger Allan, "Alliance To Standardize High-Performance DC-DC Converters" EDN magazine, ED Online ID \#7721, http: \www.edn.com

[2] Brian Narveson „POLA vs DOSA: Are the Power Module Alliances Really Different?" Power Electronics Technology, http://www.powerelectronics.com/, Apr 1, 2005

[3] J. A. Oliver, R. Prieto, V. Romero, and J. A. Cobos, "Behavioral modeling of dc-dc converters for large-signal simulation of distributed power systems," IEEE Applied Power Electronics Conference and Exposition, APEC 2006.

[4] J. A. Oliver, R. Prieto, V. Romero, and J. A. Cobos, "Behavioral Modeling of Multi-Output DC-DC Converters for Large-Signal Simulation of Distributed Power Systems," Power Electronics Specialists Conference, 2006.

[5] L. Amedo, R. Burgos, F. Wang, and D. Boroyevich, "Black-Box Terminal Characterization Modeling of DC-to-DC Converters," Applied Power Electronics Conference, APEC 2007.

[6] R. A. B. DeCarlo, M.S.; Pettersson, S.; Lennartson, B., "Perspectives and results on the stability and stabilizability of hybrid systems," Proceedings of the IEEE, vol. 88, pp. 1069-1082, 2000.

[7] LAMBDA, "Lambda X-Series Application Manual," LAMBDA 2002. 